\title{
Shelf Space Management When Demand Depends on the Inventory Level
}

\author{
Opher Baron, Oded Berman \\ Rotman School of Management, University of Toronto, Toronto, Ontario, Canada M5S 3E6, \\ Opher.Baron@Rotman.utoronto.ca, Berman@Rotman.utoronto.ca \\ David Perry \\ Department of Statistics, University of Haifa, Haifa, Israel 31905, Dperry@stat.haifa.ac.il
}

\begin{abstract}
$\mathrm{T}$ wo factors that their influence on the demand has been investigated in many papers are (i) the shelf space allocated to a product and to its complement or supplement products and (ii) the instantaneous inventory level seen by customers. Here we analyze the joint shelf space allocation and inventory decisions for multiple items with demand that depends on both factors. The traditional approach to solve inventory models with a state-dependent demand rate uses a time domain approach. However, this approach often does not lead to closed-form expressions for the profit rate with both dependencies. We analyze the problem in the inventory domain via level crossing theory. This approach leads to closed-form expressions for a large set of demand rate functions exhibiting both dependencies. These closed-form expressions substantially simplify the search for optimal solutions; thus we use them to solve the joint inventory control and shelf space allocation problem. We consider examples with two products to investigate the significance of capturing both demand dependencies. We show that in some settings it is important to capture both dependencies. We consider two heuristics, each one of them ignores one of the two dependencies. Using these heuristics it seems that ignoring the dependency on the shelf space might be less harmful than ignoring the dependency on the inventory level, which, based on computational results, can lead to profit losses of more than $6 \%$. We demonstrate that retailers should use their operational control, e.g., reorder point, to promote higher demand products.
\end{abstract}

Key words: shelf space allocation; inventory control; observed inventory level; demand dependencies; level crossing theory History: Received: January 2009; Accepted: June 2010 by Suresh Sethi, after 2 revisions.

\section{Introduction}

Improving profitability in many businesses is tied to wise demand management. One of the critical factors that influence the demand is the pricing policy. Thus, in recent years, the use of revenue management tools, namely, the control of demand via pricing, has attracted a great deal of attention from both practitioners and academics. However, demand often depends on additional factors. Two such factors that their significance has been discussed in the literature are: (i) the shelf space allocated to a product and to its complement or supplement products; and (ii) the instantaneous inventory level seen by customers. However, most of the literature considered only one of the factors above in its analysis. In this paper, we consider the joint shelf space allocation and inventory control problems when demand depends on both factors.

The effect of shelf space allocation on demand was first discussed in Lee (1961). Since then shelf space allocation has attracted a great deal of research. This research is both empirical, trying to characterize the dependency of demand on the shelf space (e.g., Desmet and Renaudin 1998) and theoretical, aiming at optimally choosing products' assortments (e.g., Akcay and Tan 2008, Aydin and Hausman 2009, Zhaolin 2007), and their shelf space allocation (e.g., Corstjens and Doyle 1981). Corstjens and Doyle (1981) also discuss how to estimate the parameters in their demand model that has been used in much of the subsequent work. For example, Bultez and Naert (1988) used it to develop a shelf space allocation model and implement it in European supermarket chains. Another optimization model and case study is presented in Borin et al. (1994). Bookbinder and Zarour (2001) introduced a non-linear programming approach to solve a two-product example. A largescale optimization model for shelf space allocation was developed by Lim et al. (2004) and Hwang et al. (2005) extended the Corstjens and Doyle's (1981) model to consider demand that depends also on the visibility of shelves. Recently, Martinez-de-Albeniz and Roels (2010) used the same model to analyze supply chain coordination.

Another optimization model for shelf space allocation was developed in van Nierop et al. (2008). They used a hierarchical Bayesian model to capture the dependency of the demand on the shelf space and its allocation and then used a lengthy simulation to optimize the shelf allocation of canned soup in a 
supermarket chain in Chicago. One of their findings is that in addition to the shelf space itself the exact location on the shelf, e.g., shelf height and shelf location within the aisle, often affect the demand. This is in line with the results reported in Lim et al. (2004) and Hwang et al. (2005).

There are two important takeaways from these papers. First, in all the empirical studies, the price fluctuation of the products was not considered a part of the control of the shelf allocation. One reason for this is that the pricing and shelf allocation decisions are taken by different levels within the organization (i.e., pricing is typically decided at the chain level whereas the allocation is often made at the store level). Another reason is that the number of changes in pricing for many common items is not sufficiently frequent to measure its effect with a significant statistical level. Therefore, we focus on a demand that is independent of price. Still, our methodology and results are easily extended to include a price-dependent demand component, for example, as in Smith and Achabal (1998).

A second takeaway from these papers is that the total shelf space is not the only factor observed by the customer that influences the demand. While the empirical component of this literature considered some additional factors (e.g., distance from the center of the aisle), it ignored others because collecting data on them is hard and incorporating them into an optimization module leads to an intractable formulation. For example, van Nierop et al. (2008) state that for these reasons they ignore the demand dependency on the shelves' layout. One additional factor that influences the demand and is ignored by the shelf space literature, possibly for the above reasons, is the level of inventory seen by customers. In the rest of the paper, when using the term inventory level we mean the inventory observed by customers.

The earliest references we are aware of discussing the dependency of demand on the inventory level is Whitin (1957). Wolfe (1968) provides empirical evidence for such dependency. The comprehensive survey by Urban (2005) that lists 60 papers on inventory-dependent demand rates shows the growing interest in such models. Balakrishnan et al. (2004, 2005) develop and implement a theoretical model that considers optimal inventory decisions in the presence of such dependency. They also cite further references on the impact of inventory level on demand. The combined influence of pricing and the inventory level on demand was investigated by Smith and Achabal (1998), and supply chain coordination when such dependency exists was studied in Wang and Gerchak (2001). Periodic review models with demand that depends on the initial inventory level was studied by Gerchak and Wang (1994).
Most of the papers that discuss shelf space ignore inventory-related cost and the influence of the inventory level on the demand. An exception that considers the inventory cost is Hwang et al. (2005) who confine the shelf space to equal the reorder point plus the order quantity. To the best of our knowledge, the only paper that considers both demand dependencies is Hariga et al. (2007). They focused on formulating a detailed optimization model. We, in contrast, focus on the implication of the joint dependency and provide managerial insights on its importance. Furthermore, we consider a substantially more general demand model than theirs. For example, their demand rate model includes no intercept, constraining the demand at zero inventory level to equal zero. However, in many stores there is a base demand that is independent of the inventory level. For example, on page 971, Datta and Pal (1990) write "... customers arrive to purchase goods owing to such factors as goodwill, good quality ...." Thus, as noted by Balakrishnan et al. (2004), imposing zero demand at zero inventory level is often unrealistic.

An empirical evidence for the joint dependency of demand on the shelf space and the inventory level has been demonstrated in Parker and Lehmann (2009). They supported and extended the empirical work of van Herpen et al. (2009) on the bandwagon effect. This effect suggests that products with lower inventory level, i.e., partially empty shelf space, may present higher demand than if the shelf is full. Specifically, Parker and Lehmann (2009) tested the joint influence of the shelf space allocated and the inventory level on the demand. In their Experiment 3a (Parker and Lehmann 2009), they exposed participants to shelves with two brands of wine, with several different levels of inventory depletion and with one brand having higher allocated shelf space. They concluded that "the wine given more shelf space was significantly more preferred when it was the scarcer alternative." This observation implies that it is important to consider both the allocated shelf space and the inventory level when estimating the demand. In such cases our model is useful. While the empirical tests in both van Herpen et al. (2009) and Parker and Lehmann (2009) considered demand for wines, additional examples where a joint dependency is probably observed in practice are easy to come up with, including the canned soup category that the dependence of its demand on the shelf space allocation has been addressed before, e.g., van Nierop et al. (2008) and references within. More recently, additional empirical evidence for the influence of inventory level and shelf space on demand is given by Ton and Raman (2010) who focus on the influence of total sales of products that are only available in the back room (rather than on the shelf).

One of the main objectives of this paper is to investigate whether there is a practical importance in 
capturing the demand dependency on both the inventory level and the shelf space allocation. For this, we analyze the shelf space allocation and inventory decisions for multiple items when demand exhibits both dependencies. We develop a closed-form expression for the profit rate of a retailer selling several products competing over shelf space when each product's demand depends on both its inventory level and the shelf space allocated to all products. Using this closed form, we can solve the joint inventory control (reorder points and order quantities) and the shelf space allocation problems to maximize the expected profit rate of retailers. We consider examples with two products competing over shelf space. We conclude that, in some settings, it is important to capture both demand dependencies. We find that ignoring the dependency on the inventory level might be more harmful than ignoring the dependency on the shelf space and can lead to profit losses of more than $6 \%$. We demonstrate that ignoring the intercepts in the demand model might be very costly. We also investigate how do the optimal decision variables and profit change with different parameters of the demand. Our main conclusion is that in the presence of the demand dependency on the shelf space and the inventory level retailers should use their operational controls in order to promote higher demand products.

The traditional approach for solving the inventorydependent models uses the time domain to generate a differential equation that expresses the inventory level during the cycle, e.g., Baker and Urban (1988). However, solving the differential equation is possible only for very limited demand rate functions, e.g., the power function (Urban 2005). Moreover, we will show in section 2.2 that this differential equation may not be solved when dependency on the shelf space is added. Thus, the time domain methodology leads to tractable models only in restricted settings. It is important to note that the traditional approach fails to provide closed-form expressions even when the dependency on the shelf space is ignored for demand rates that include an intercept. This shows the weakness of the traditional approach.

Our methodology for the analysis of inventorydependent demand rate is different and is another contribution. Rather than analyzing the model via the time domain - the horizontal axis, we transform the model to the inventory domain-the vertical axis in the traditional approach. To establish this methodology we use level crossing theory (e.g., Cohen 1977). We show that the inventory domain approach leads to closed-form expressions and that it is superior to the time domain approach in many settings. It is important to note that the methodology of the inventory domain approach may be applicable in other settings where the time domain approach fails. This can be an interesting future research direction.
Obtaining a closed-form expression for the profit rate is important because, in addition to enabling a substantial reduction in the effort and computation time of our optimization model, they allow for sensitivity analysis of various parameters and an investigation of the asymptotic behavior of the solution. These can lead to important managerial implications. For example, in section 4.1, using sensitivity analysis, we show that order quantities and allocated shelf space are independent of the markup and that the reorder points are increasing with it. In the same section, we provide the asymptotic behavior of the profit when the shelf space increases. We emphasize that without the closed-form expressions, obtained using the time domain approach, deriving such results would be extremely difficult.

The paper is organized as follows: In section 2, we introduce a general model and demonstrate the failure of the time domain approach for a specific demand rate function. Then, in section 3, we use level crossing theory to transform the problem to the inventory domain. We then demonstrate the use of these results for a single item model assuming some common demand dependencies. In section 4, we discuss several heuristics, present numerical study of two items competing over shelf space, and derive some useful managerial insights. Conclusions and suggestions for future research are given in section 5 .

\section{The Model}

In this section, we present models for inventory management aiming at profit maximization when demand depends on both the inventory level and the shelf space. We demonstrate the difficulty in using the traditional time domain approach for maximizing the profit in a simple example. In section 3, we analyze the same example, showing that using the inventory domain approach it is tractable.

Table of Frequently Used Notation

$$
\begin{aligned}
& N \quad=\text { Number of products } \\
& S_{T} \quad=\text { Shelf space available } \\
& S_{i} \quad=\text { Shelf space allocated to product } i \text {, } \\
& i=1,2, \ldots, N\left(\vec{S}=\left(S_{1}, S_{2}, \ldots, S_{N}\right)\right) \\
& t=\text { Time } \\
& I_{i}(t, \vec{S}) \quad=\text { The inventory level of product } i \text { at time } t \\
& r_{i}\left(I_{i}(t, \vec{S}), \vec{S}\right)=\text { The demand rate for product } i \\
& c_{i} \quad=\text { Unit cost for product } i \\
& p_{i} \quad=\text { Markup of product } i \\
& a_{i} \quad=\text { Re-order point for product } i \\
& q_{i} \quad=\text { Order quantity for product } i \\
& T_{i} \quad=\text { Cycle length for product } i \\
& K_{i} \quad=\text { Set-up cost for product } i
\end{aligned}
$$




$$
\begin{aligned}
h_{i}\left(I_{i}(t, \vec{S})\right) d t= & \text { The infinitesimal holding cost function } \\
& \text { for product } i \\
H_{i} & =\text { Total holding cost per cycle for product } i \\
c_{S_{i}} & =\text { The difference per time unit between the } \\
& \text { cost of stocking product } i \text { on the shelf } \\
& \text { and the payment received for this space } \\
f(I) \quad & =\text { Probability density function of the in- } \\
& \text { ventory level }
\end{aligned}
$$

\subsection{The Inventory and Demand Rates Modeled}

Consider a retailer that manages inventory and shelf allocation of $N$ products over a total shelf space, $S_{T}$. We let $\vec{S}$ be the vector of shelf space allocation with $S_{i} \geq 0$ being the shelf space allocated to product $i=1, \ldots, N$; thus, $\sum_{i=1}^{N} S_{i} \leq S_{T}$. The demand for item $i$ depends on the entire shelf space allocation. This is true not only because all products share a restricted shelf space, but also because there might be some cross shelf space relations between the demands for different items. We allow the demand rate for product $i$ at time $t$ to depend on both the shelf space vector, $\vec{S}$, and the inventory level of this product at time $t, I_{i}(t, \vec{S})$ (observe that the inventory level at time $t$ also depends on $\vec{S}$ ). We denote this demand rate by $r_{i}\left(I_{i}(t, \vec{S}), \vec{S}\right)$. To simplify the notation we use $I_{i}(t, \vec{S})=$ $I_{i}$ when no confusion exists.

We assume that the demand rates, $r_{i}\left(I_{i}, \vec{S}\right)$, for each $i=1, \ldots, N$ are continuous deterministic functions $0 \leq r_{i}\left(I_{i}, \vec{S}\right)<\infty, \forall \vec{S} \geq 0$ such that $\sum_{i=1}^{N} S_{i} \leq S_{T}$. Then if $r_{i}\left(I_{i}, \vec{S}\right)>0$ for any $I_{i}^{1} \leq I_{i} \leq I_{i}^{2}$ such that $0 \leq I_{i}^{1} \leq$ $I_{i}^{2}<\infty$ we have

$$
\begin{aligned}
0< & \int_{I_{i}^{1}}^{I_{i}^{2}} \frac{1}{r_{i}\left(I_{i}, \vec{S}\right)} d I_{i}<\infty \quad \forall \vec{S} \geq 0, \quad \text { such that } \\
& \sum_{i=1}^{N} S_{i} \leq S_{T},
\end{aligned}
$$

representing the time required for the inventory level of item $i$ to move from state $I_{i}^{2}$ down to state $I_{i}^{1}$ with no orders arriving in between. We consider an infinite horizon inventory model. Let the unit cost of product $i$ be $c_{i}$ and its sale price be $c_{i} p_{i}$, where $p_{i}$ is the markup (of course we allow $c_{i}$ and $p_{i}$ to differ across products). Thus, ignoring the operational costs, the profit per unit of product $i$ that is sold is $c_{i}\left(p_{i}-1\right)=c_{i} p_{i}-c_{i}$. We assume that each product $i$ can be ordered with its own reorder point, $a_{i}$, and order quantity, $q_{i}$. We describe the model assuming a zero lead time (extending the analysis to a positive deterministic lead time is possible) as follows: For each product $i$, whenever the inventory level $I_{i}(t, \vec{S})$ drops to a level $a_{i} \geq 0$, an order of size $q_{i}>0$ is placed so that $S_{i} \leq a_{i}+q_{i}$. For simplicity, we assume that, when the inventory level is higher than the shelf space, $S_{i}$, the excess inventory is used to instantaneously replenish the shelf. This has been also assumed by Hwang et al. (2005) and Hariga et al. (2007). (The analysis when the shelf space is not instantaneously filled requires specific assumptions on the restocking process, but is similar.) Thus, as long as $I_{i} \geq S_{i}$ the inventory level seen by customers is $S_{i}$. As a result, during each cycle the demand rate has two parts, the first depends only on the shelf space allocation vector (whenever the inventory level is above $S_{i}$ ), and the second depends on both the shelf space allocated to other products and the inventory level (when the latter is between $a_{i}$ and $S_{i}$ ).

As a mathematical trick we assume, for the time being, that, when ordering $q_{i}$ products, only $Y_{q i}$ of them are good, where $Y_{q i}$ is a random variable with a cumulative distribution $F_{Y_{q_{i}}}\left(y_{q_{i}}\right)$, e.g., Henig and Gerchak (1988). Eventually, the results are specialized to the more relevant case where $Y_{q_{i}}=q$.

For all $a_{i}+q_{i}$ and $\vec{S}$, the inventory level process $I_{i}(t$, $\vec{S})$ is a regenerative process where the time between arrival of orders is the cycle length, $T_{i}$. This is true because we assumed that the dependency of product $i$ 's demand on the other products is captured only through the shelf space allocated to them and not through their actual inventory levels. In principle, demand for an item might also depend on the inventory levels of other items. (An alternative formulation assuring that the inventory level processes are regenerative is to restrict all cycle lengths to be identical. We do not follow this alternative.) However, for tractability purposes and because in many cases the shelves are full, we only allow cross items demand dependencies via the shelf size. The same assumption is also made by Hariga et al. (2007).

For a reorder point $a_{i}$ and a yield $Y_{q_{i}}$, the length of a cycle $T_{i}$, is

$$
\int_{a_{i}}^{a_{i}+Y_{q_{i}}} \frac{1}{r_{i}\left(I_{i}, \vec{S}\right)} d I_{i}
$$

Thus,

$$
E\left(T_{i}\right) \equiv E\left(T_{i}\left(a_{i}, q_{i}, \vec{S}\right)\right)=E\left(\int_{a_{i}}^{a_{i}+Y_{q_{i}}} \frac{1}{r\left(I_{i}, \vec{S}\right)} d I_{i}\right)
$$

is the expected length of a cycle. We assume that shortages are not allowed and whenever a new order is placed a set-up cost of $K_{i}$ is incurred.

Let $h_{i}\left(I_{i}(t, \vec{S})\right) d t>0$ be the infinitesimal holding cost function for product $i$ and let $H_{i}$ denote the total holding cost per cycle for this product. Then, letting $t=0$ at the beginning of the cycle, the expectation of $H_{i}$ is

$$
E\left(H_{i}\right)=E\left(\int_{0}^{T_{i}\left(a_{i}, q_{i}, \vec{S}\right)} h_{i}\left(I_{i}(t, \vec{S})\right) I_{i}(t, \vec{S}) d t\right)
$$


(for notational convenience we omit the dependency of $E\left(H_{i}\right)$ on $\vec{S}, a_{i}$, and $\left.q_{i}\right)$. Note that (4) assumes that the holding costs of items in the backroom (when the total inventory of product $i$ held by the retailer is larger than $S_{i}$ ) and on the shelves are identical, as is typical in retailer environments. A simple modification is possible when these holding costs differ.

To simplify the exposition, we model the difference between the cost for stocking product $i$ on the shelf and the payment received for this space from the wholesaler in a linear fashion. We denote this difference per time unit by $c_{s_{i}}$ (note that we do not restrict the sign of $\left.c_{s_{i}}\right)$. Note that $c_{i}\left(p_{i}-1\right) E\left(Y_{q_{i}}\right)$ is the expected proceeds obtained from sales over a cycle. Thus, the long-run profit rate, $R_{i}\left(a_{i}, q_{i}, \vec{S}\right)$, is

$$
R_{i}\left(a_{i}, q_{i}, \vec{S}\right)=\frac{c_{i}\left(p_{i}-1\right) E\left(Y_{q_{i}}\right)-E\left(H_{i}\right)-K_{i}}{E\left(T_{i}\right)}+c_{S_{i}} S_{i} .
$$

Thus, the retailer faces the following optimization problem

$$
\begin{gathered}
\max _{\vec{a}, \vec{q}, \vec{S}} \sum_{i=1}^{N} R_{i}\left(a_{i}, q_{i}, \vec{S}\right), \\
\sum_{i=1}^{N} S_{i} \leq S_{T} \\
0 \leq a_{i} \leq S_{i} \leq a_{i}+q_{i} \quad \forall i=1, \ldots, N .
\end{gathered}
$$

We note that if $c_{s_{i}} \geq 0$ and $S_{T}=\infty$, there is no reason to limit the shelf space allocated for products. Thus, $S_{i}=a_{i}+q_{i}$. We further note that constraints on the minimal or maximal shelf space or order quantity per item, as was used in Corstjens and Doyle (1981), can also be incorporated in this optimization problem. However, for simplicity of the exposition, we ignore them. Finally, due to the high value of shelf space in practice, we assume that it is never optimal to have $S_{i}>a_{i}+q_{i}$; however, this constraint is not essential.

\subsection{Specific Demand Rate Forms}

The most widely used demand rate model that depends on shelf space is the one due to Corstjens and Doyle (1981). Letting $d_{i}(\vec{S})$ denote the total demand over a cycle, given shelf space allocation $\vec{S}$, they model

$$
d_{i}(\vec{S})=\hat{\alpha}^{i} S_{i}^{\beta_{i}} \prod_{\substack{j=1 \\ j \neq i}}^{N} S_{j}^{\delta_{i j}}
$$

where $\hat{\alpha}^{i}>0$ is a scaling constant, $\beta_{i}$ is the direct elasticity with respect to a unit of shelf space, and $\delta_{i j}$ are the cross space elasticities between products $i$ and $j$.

If the cycle length for the $i$ th item is $T_{i}$, from (8) we have the following demand rate:

$$
\hat{r}_{i}(\vec{S})=\frac{\hat{\alpha}^{i} S_{i}^{\beta_{i}} \prod_{\substack{j=1 \\ j \neq i}}^{N} S_{j}^{\delta_{i j}}}{T_{i}} .
$$

However, by letting $\alpha^{i}=\hat{\alpha}^{i} / T_{i}$ without loss of generality, the demand rate is

$$
r_{i}(\vec{S})=\alpha^{i} S_{i}^{\beta_{i}} \prod_{\substack{j=1 \\ j \neq i}}^{N} S_{j}^{\delta_{i j}} .
$$

Then, by (2) $T_{i}$ is given by

$$
T_{i}=\int_{a_{i}}^{a_{i}+Y_{q_{i}}} \frac{d x}{r_{i}(\vec{S})} .
$$

Corstjens and Doyle's (1981) model ignores the demand dependency on the inventory level and the inventory-related cost. The survey of Urban (2005) lists a few demand rate functions that capture such dependency. In that literature the focus was on a single item and the most common demand rate is the multiplicative (e.g., Baker and Urban (1988):

$$
r_{i}\left(I_{i}\right)=\alpha I_{i}^{\beta},
$$

where $\alpha>0$ and $\beta \in[0,1)$. As pointed out in Balakrishnan et al. (2004) with this demand rate function the demand is zero when the inventory is zero, which is restrictive in many cases. A natural way to extend (10) to include a positive demand rate when no inventory is held is by adding an intercept $\alpha_{0}$

$$
r_{i}\left(I_{i}\right)=\alpha_{0}+\alpha_{2} I_{i}^{\beta} .
$$

The standard approach to treat inventory problems with inventory-dependent demand rate such as (10) and (11) uses the time domain. However, as we show next, this approach fails to express the profit rate $R(a, q, S)$ in a closed form even for a single product in many settings. The example of the failure of time domain approach below includes the case when $r(I)$ is given by (11).

Example 1. Power Demand Rate-Failure of the Time Domain Approach: To demonstrate the time domain approach and why it fails in more general settings, we consider the power demand rate

$$
r(I, S)= \begin{cases}\alpha_{0}+\alpha_{1} S^{\beta_{1}}+\alpha_{2} S^{\beta_{2}} & I \geq S \\ \alpha_{0}+\alpha_{1} S^{\beta_{1}}+\alpha_{2} I^{\beta_{2}} & 0 \leq I<S,\end{cases}
$$

with $\alpha_{0}, \alpha_{1}, \alpha_{2} \geq 0$ and where $\beta_{1} \in[0,1)$ captures the demand rate dependency on the shelf space and $\beta_{2} \in[0,1)$ captures the demand rate dependency on the inventory level.

The time domain approach for solving the problem requires the solution of two differential equations to be able to calculate $E(T)$ and $E(H)$. The differential equation for the inventory at time $t, I(t) \in(a, S)$ is

$$
I(t)=S-\int_{0}^{t} \alpha_{0}+\alpha_{1} S^{\beta_{1}}+\alpha_{2} I(v)^{\beta_{2}} d v .
$$


However, we could not solve (13) in a closed form. This is in agreement with Urban (2005) who mentions this difficulty in solving the differential equation for $I(t)$. It should be noted that even when $\alpha_{1}=0$ as in (11), i.e., the demand rate is independent of the shelf space, there is no solution to (13) and thus the classical approach to solve the problem cannot be carried out (when both $\alpha_{0}=\alpha_{1}=0$, as in Hariga et al. (2007), (13) can be solved; thus the time domain approach is tractable in that case).

To summarize, this example demonstrates the difficulty of the time domain approach in obtaining $E(H)$ and $E(T)$ that are required for optimization purposes. While (13) may be solved numerically, it is not easily amenable for optimization. In theory, an optimization program that searches for optimal reorder point, quantity to order, and shelf space level could pursue an exhaustive search over different combinations of these parameters while solving (13) numerically for different parameter combinations. However, once the number of items increase and so does the number of decision variables, such an exhaustive search is not practical. We suspect that this limitation prevents researchers from considering (11), (12), and other demand rate functions.

Our purpose is to allow the demand rate to depend on both the shelf space and the observed inventory level. More importantly, we investigate whether it is desirable to accurately capture this joint dependency. To achieve this we consider a demand rate combining both the shelf space dependence, as in (9), with the inventory level dependence, as in (11). To represent the demand rate for the $i$ th product, we add a subscript $i$ to the parameters in (12); we also let $\delta_{1 i j}, \in(-1,1)$ capture the dependency between products $i$ and $j$ as they affect the $i$ th product's shelf space; and $\delta_{2 i j}, \in(-1,1) j \neq i$, capture the dependency between products $i$ and $j$ as they affect the $i$ th item's inventory level ${ }^{1}$ :

$$
\begin{aligned}
& r_{i}\left(I_{i}, \vec{S}\right)= \\
& \begin{cases}\alpha_{0 i}+\alpha_{1 i} S_{i}^{\beta_{1 i}} \Pi_{j \neq i} S_{j}^{\delta_{1 i j}}+\alpha_{2 i} S_{i}^{\beta_{2 i}} \Pi_{j \neq i} S_{j}^{\delta_{2 i j}} & I_{i} \geq S_{i} \\
\alpha_{0 i}+\alpha_{1 i} S_{i}^{\beta_{1 i}} \Pi_{j \neq i} S_{j}^{\delta_{1 i j}}+\alpha_{2 i} I_{i}^{\beta_{2} i} \Pi_{j \neq i} S_{j}^{\delta_{2 i j}} & 0 \leq I_{i}<S_{i} .\end{cases}
\end{aligned}
$$

Note that, in accordance with our assumption that the shelf space is instantaneously filled whenever $I_{i}>S_{i}$, the demand rate function (14) has two parts. The first is for $I_{i} \geq S_{i}$, where both the observed inventory equals the shelf space and their effect is captured only by $S_{i}$, and the second is for $I_{i}<S_{i}$, where the inventory level dependency is affected by $I_{i}$ and the shelf space dependency is affected by $S_{i}$.
Notice that when $\alpha_{1 i}=\alpha_{2 i}=0, r_{i}\left(I_{i}, \vec{S}\right)$ is just the demand rate in the standard EOQ model, when $\alpha_{0 i}=\alpha_{1 i}=\delta_{2 i j}=0, r\left(I_{i}, S\right)$ is the power demand rate given in (10), and when $\alpha_{0 i}=\alpha_{2 i}=0$ the demand rate depends only on the shelf space as in (9).

As the demand rate function in (14) is a generalization of the one in (12) that as shown above is not tractable using the time domain approach, we next develop the profit function in an alternate manner via the inventory theory domain approach.

\section{The Inventory Domain-Analysis Using Level Crossing}

To analyze the long-run average costs in the inventory domain, we focus on the profit generated by a single product, thus in this section we suppress the subscripts when no confusion occurs. We use level crossing theory (e.g., Cohen 1977). Using level crossing theory is possible because the inventory process is regenerative. Observe that, when time is measured from the beginning of a cycle, knowing the jump size at $t=0$ results in a deterministic demand rate. Therefore, the inventory level within a cycle is a monotone function and knowing $t$ reveals the inventory level $I$ and vice versa. This simplifies the analysis of the problem using the inventory domain approach.

To further motivate the inventory domain approach we observe that if a continuous probability density function for the inventory level, $f(I)$, exists, the holding cost rate would be $\int_{a}^{\infty} h(I) I f(I) d I$. Level crossing theory shows how to obtain this distribution and that it exists when the model is not deterministic. If the model is completely deterministic, $f(I)$ has the meaning of time average. Below, we provide an intuitive explanation of this approach.

To express $f(I)$, we equate the rate of upcrossings and downcrossings of each inventory level I. Observe that the inventory level can only decrease below $I$ after the inventory level jumped above $I$, due to a replenishment, and vice versa. Therefore, in each cycle the inventory level upcrosses and downcrosses level $I \in(a, a+q)$ at most once. In fact, each $I$ will be upcrossed in a cycle if the delivered quantity, $Y_{q}$, is larger than $I-a$. Thus, the expected number of upcrossings $I$ in a cycle is $1-F_{Y_{q}}(I-a)$. To calculate the expected number of downcrossings, we can view $f(I)$ as the proportion of time that inventory level at time $t$, $I(t) \in d I$ during a single time unit. Therefore, in a cycle whose expected length is $E(T)$ the average time $I(t) \in d I$ is $E(T) f(I)$. By Little's law, we have

$$
\begin{aligned}
& E(\text { number of downcrossings of } I \text { in a cycle) } \\
& \quad=E(T) f(I) r(I, \vec{S}),
\end{aligned}
$$

because $r(I, \vec{S})$ is the demand rate. Because the number of upcrossings and downcrossings are equal, we 
obtain $E(T) f(I) r(I, \vec{S})=1-F_{Y_{q}}(I-a)$ so that

$$
f(I)=\left\{\begin{array}{cl}
\frac{1-F_{Y_{q}}(I-a)}{r(I, \widehat{S}) E(T)} & \text { for } I \geq a \\
0 & \text { otherwise. }
\end{array}\right.
$$

Note that (15) is a simple generalization of Theorem 1 in Berman and Perry (2006). They did not consider dependency of the demand on the shelf space. Observe that to ensure that (15) is a proper probability density function we must have

$$
E(T)=\int_{a}^{\infty} \frac{1-F_{Y_{q}}(I-a)}{r(I, \vec{S})} d I,
$$

giving an alternative representation for $E(T)$ in (3).

Therefore, in cases that $f(I)$ exists (or when it represents the time average) the holding cost rate is

$$
\frac{E(H)}{E(T)}=\int_{a}^{\infty} h(I) I f(I) d I .
$$

Thus substituting (16) and (17) into (5), we obtain for the $i$ th item.

THEOREM 1. For continuous demand rates satisfying (1) the profit rate for the ith item is given by

$$
\begin{aligned}
R\left(a_{i}, q_{i}, \vec{S}\right)= & \frac{c_{i}\left(p_{i}-1\right) E\left(Y_{q_{i}}\right)-K_{i}-\int_{a_{i}}^{\infty} h_{i}(I) I \frac{1-F_{Y_{q_{i}}}\left(I-a_{i}\right)}{r_{i}(I, \vec{S})} d I}{\int_{a_{i}}^{\infty} \frac{1-F_{Y_{q_{i}}}\left(I-a_{i}\right)}{r_{i}(I, \vec{S})} d I} \\
& +c_{s_{i}} S_{i} .
\end{aligned}
$$

The rigorous proof of Theorem 1 is given in Appendix A using level crossing theory. We note that this proof carries forward even when adding a multiplicative dependency in price, as in Smith and Achabal (1998).

OBSERVATION 1. When $Y_{q_{i}}=q_{i}$, i.e., when setting $F_{Y_{q_{i}}}(I)=$ 0 for each $I<q_{i}$ and $F_{Y_{q_{i}}}(I)=1$ for each $I \geq q_{i}$, for each $i$, we obtain

$$
\begin{aligned}
R\left(a_{i}, q_{i}, \vec{S}\right)= & \frac{c_{i}\left(p_{i}-1\right) q_{i}-K_{i}-\int_{a_{i}}^{a_{i}+q_{i}} h_{i}(I) I \frac{1}{r_{i}(I, \vec{S})} d I}{\int_{a_{i}}^{a_{i}+q_{i}} \frac{1}{r_{i}(I, \vec{S})} d I} \\
& +c_{S_{i}} S_{i} .
\end{aligned}
$$

Thus, from a practical point of view, our solution approach substantially reduces the optimization time and effort when closed-form expressions for the integrals in (19) exist. We next give one such example.

Example 2. (Continuation of Example 1) Power Demand Rate-Success of the Inventory Domain Approach: To demonstrate the solution method discussed above, we consider a single item case with the power demand rate function from (12). This empha- sizes the advantage of the inventory domain approach over the time domain approach; as shown in section 2.2 , the latter leads to an intractable model in this case. While in this single product case, there is essentially no competition on the shelf space, we give the detailed analysis to demonstrate the inventory domain approach. The development for several items is similar and leads to similar closed-form expressions.

We first rewrite (16) (assuming that we always receive the full order):

$$
\begin{aligned}
E(T)= & \int_{a}^{\min \{S, a+q\}} \frac{d I}{\alpha_{0}+\alpha_{1} S^{\beta_{1}}+\alpha_{2} I^{\beta_{2}}} \\
& +\int_{S}^{\max \{S, a+q\}} \frac{d I}{\alpha_{0}+\alpha_{1} S^{\beta_{1}}+\alpha_{2} S^{\beta_{2}}} .
\end{aligned}
$$

For example, if $a+q \geq S$, as required by (7), we have

$$
\begin{aligned}
& E(T)=\frac{a+q-S}{\alpha_{0}+\alpha_{1} S^{\beta_{1}}+\alpha_{2} S^{\beta_{2}}} \\
& +\frac{S \operatorname{LerchPhi}\left(-\alpha_{2} \frac{S^{\beta_{2}} \alpha_{2} S^{\beta_{1}}}{\alpha_{0}}, 1, \frac{1}{\beta_{2}}\right)-a \operatorname{LerchPhi}\left(-a^{\beta_{2}} \frac{\alpha_{2}}{\alpha_{0}+\alpha_{1} S^{\beta_{1}}}, 1, \frac{1}{\beta_{2}}\right)}{\left(\alpha_{0}+\alpha_{1} S^{\beta_{1}}\right) \beta_{2}}
\end{aligned}
$$

where $\operatorname{LerchPhi}(z, f, r) \equiv \sum_{k=0}^{\infty} \frac{z^{k}}{(r+k)^{f}}$ (see, e.g., http:// mathworld.wolfram.com/LerchTranscent.html) and is implemented in common mathematical software. For the same example, we obtain the holding cost per cycle $E(H)$, from (17), to be

$$
\begin{aligned}
& E(H) E(T)=\frac{1}{2} h \frac{(a+q)^{2}-S^{2}}{\alpha_{0}+\alpha_{1} S^{\beta_{1}}+\alpha_{2} S^{\beta_{2}}} \\
& +h \frac{S^{2} \operatorname{LerchPhi}\left(-\alpha_{2} \frac{S^{\beta_{2}}+\alpha_{1}}{\alpha_{1} \beta_{1}}, 1, \frac{2}{\beta_{2}}\right)-a^{2} \operatorname{LerchPhi}\left(-\alpha_{2} \frac{a^{\beta_{0}}+\alpha_{1}}{\beta_{1}}, 1, \frac{2}{\beta_{2}}\right)}{\left(\alpha_{0}+\alpha_{1} S^{\beta_{1}}\right) \beta_{2}}
\end{aligned}
$$

Substituting (20) and (21) in (18) gives $R(a, q, S)$.

\section{Items Competing for Shelf Space}

We now return to the problem in (6). Using the development in sections 3 and 2.2, the expressions required to evaluate the profit in (6) can be found in closed form. This closed form can be used in conjunction with any non-linear optimization package to find the optimal shelf space allocation, reorder points, and order quantities. This is in contrast to the discussion following Example 1. Thus, our inventory domain approach considerably simplifies the optimization problem faced by the decision problem.

There are a few questions we wish to investigate. First, how do the optimal decision variables and profit change with different parameters of the demand function? Second, is there any importance in capturing both demand dependencies? Finally, is including an intercept in the demand model relevant? 
In the next subsection, we describe the numerical experiments designed to answer these questions and their results for cases of two products competing over shelf space.

\subsection{Sensitivity of Optimal Decisions to Different Settings}

To simplify the investigation in this section, we focused a special case of (14) where $\alpha_{1 i}=\alpha_{2 i}, \beta_{1 i}=\beta_{2 i}$, $\delta_{1 i j}=\delta_{2 i j}$ and we denote these coefficients by $\alpha_{i}, \beta_{i}$, and $\delta_{i j}$, respectively:

$$
r_{i}\left(I_{i}, \vec{S}\right)= \begin{cases}\alpha_{0 i}+\alpha_{i} \Pi_{j \neq i} S_{j}^{\delta_{i j}}\left(S_{i}^{\beta_{i}}+S_{i}^{\beta_{i}}\right) & I_{i} \geq S_{i} \\ \alpha_{0 i}+\alpha_{i} \Pi_{j \neq i} S_{j}^{\delta_{i j}}\left(S_{i}^{\beta_{i}}+I_{i}^{\beta_{i}}\right) & 0 \leq I_{i}<S_{i} .\end{cases}
$$

This reduces the number of parameters needed to be considered.

For our numerical examples $c_{1}=c_{2}=1, K_{1}=K_{2}=$ $10, h_{1}=h_{2}=0.05, c_{s_{1}}=c_{s_{2}}=0$. We let $\alpha_{01}=\alpha_{02}=1$, $\alpha_{1}=15$, and $\alpha_{2}=10$, so that product 1 is the product with the higher demand. We consider three types of shelf space and inventory influence: high, where $\beta_{1}=\beta_{2}=0.7$ and $\delta_{12}=\delta_{21}=0.2 ;$ medium, where $\beta_{1}=$ $\beta_{2}=0.5$ and $\delta_{12}=\delta_{21}=0.1$; and low, where $\beta_{1}=\beta_{2}=$ 0.3 and $\delta_{12}=\delta_{21}=0.05$. We name these types the high-, medium-, and low-influence types, respectively. In all these types we choose the markup $p_{1}=p_{2}=1.3$. We note that these values for $\beta^{\prime}$ s and $\delta^{\prime}$ s agree with Bultez and Naert (1988) and Desmet and Renaudin (1998) stating that the influence of other products' shelf space is lower than that of the product's shelf space and that similar $\beta$ values are found in practice.

In Table 1, we present the ratio of the profit, reorder points, order quantities, and shelf space allocated when $S_{T}$ is increasing, from 50 to 1000 . For example, for the low-, medium-, and high-influence types, the profit increases by 2.96, 6.8, and 16.73, while the optimal shelf space allocated for each product increases by about 20 times. As expected, the increase in total shelf space is more beneficial when the demand influence is higher.

In Tables 2-4, we present, for the high-, medium-, and low-influence types, the total allowed shelf space, $S_{T}$, and the optimal profit; reorder points, $a_{1}$ and $a_{2}$; order quantities, $q_{1}$ and $q_{2}$; and shelf space allocated to products, $S_{1}$ and $S_{2}$. We varied the shelf space

Table 1 Ratios of Optimal Profits and Controls as $S_{T}$ is Increasing from 50 to 1000

\begin{tabular}{lrcccccc}
\hline & Profit & $a_{1}$ & $a_{2}$ & $q_{1}$ & $q_{2}$ & $S_{1}$ & $S_{2}$ \\
\hline Low influence & 2.96 & 31.65 & 308.1 & 2.75 & 2.65 & 19.53 & 20.69 \\
Medium influence & 6.80 & 42.86 & 69.86 & 2.73 & 2.81 & 19.79 & 20.32 \\
High influence & 16.73 & 29.41 & 37.94 & 3.41 & 3.46 & 19.89 & 20.15 \\
\hline
\end{tabular}

Table 2 Sensitivity to Different Total Shelf Space: High-Influence Type

\begin{tabular}{lrrrrrrr}
\hline$S_{T}$ & \multicolumn{1}{c}{ Profit } & \multicolumn{1}{c}{$a_{1}$} & \multicolumn{1}{c}{$a_{2}$} & \multicolumn{1}{c}{$q_{1}$} & \multicolumn{1}{c}{$q_{2}$} & \multicolumn{1}{c}{$S_{1}$} & \multicolumn{1}{c}{$S_{2}$} \\
\hline 50 & 230.02 & 17.63 & 9.74 & 527.66 & 402.57 & 28.97 & 21.03 \\
75 & 341.19 & 29.83 & 18.48 & 579.89 & 448.53 & 43.39 & 31.61 \\
100 & 449.56 & 42.50 & 27.47 & 632.84 & 486.95 & 57.83 & 42.17 \\
150 & 660.74 & 67.49 & 44.73 & 760.07 & 585.87 & 86.67 & 63.33 \\
200 & 866.39 & 90.37 & 60.60 & 893.06 & 685.59 & 115.45 & 84.55 \\
350 & 1461.01 & 169.30 & 115.98 & 1165.31 & 901.57 & 201.94 & 148.06 \\
500 & 2033.19 & 249.67 & 174.87 & 1308.97 & 1014.19 & 288.31 & 211.69 \\
1000 & 3848.53 & 518.55 & 369.61 & 1800.80 & 1393.44 & 576.15 & 423.85 \\
5000 & $16,701.06$ & 2734.98 & 1978.70 & 3900.88 & 2966.25 & 2879.87 & 2120.13 \\
10,000 & $31,296.53$ & 5545.62 & 4055.47 & 5336.26 & 4060.17 & 5758.45 & 4241.55 \\
\hline
\end{tabular}

between 50 and 10,000 in different intervals. For the medium- and high-influence types, we present the results for 10 values of the total shelf space $S_{T} \in\{50,75,100,150,200,350,500,1000,5000,10,000\}$. For the low-influence type, it turns out that a total shelf space of 2497 maximizes the profit. Thus, for this type we present 11 values of $S_{T} \in\{50,75,100,150,200,350$, 500, 1000, 1500, 2000, 2497\}. From these tables we find:

1. As expected, for all three types the profit, order quantities, and shelf space allocated to items are increasing with the total shelf space. Other observations are that ratios $a_{1} / a_{2}, S_{1} / S_{2}$, and $q_{1} /$ $q_{2}$ are all larger than 1 . Thus, the retailer gains an operational benefit by using her controls to promote the higher demand product. In addition,

(a) For the high- and medium-influence types, we observed that

- the reorder points are increasing with the shelf space. Thus, the higher demand rate implied by the higher allocated shelf space induces the retailer to carry more items during the whole cycle. This means that for

Table 3 Sensitivity to Different Total Shelf Space: Medium-Influence Type

\begin{tabular}{lrrrrrrr}
\hline$S_{T}$ & \multicolumn{1}{c}{ Profit } & \multicolumn{1}{c}{$a_{1}$} & \multicolumn{1}{c}{$a_{2}$} & \multicolumn{1}{c}{$q_{1}$} & \multicolumn{1}{c}{$q_{2}$} & \multicolumn{1}{c}{$S_{1}$} & \multicolumn{1}{c}{$S_{2}$} \\
\hline 50 & 77.29 & 8.57 & 3.00 & 308.47 & 232.48 & 29.84 & 20.16 \\
75 & 101.79 & 15.45 & 6.20 & 348.25 & 264.96 & 44.65 & 30.35 \\
100 & 123.30 & 22.87 & 9.89 & 381.44 & 291.17 & 59.46 & 40.54 \\
150 & 160.83 & 38.79 & 18.24 & 435.57 & 332.43 & 89.06 & 60.94 \\
200 & 193.64 & 56.09 & 27.89 & 474.59 & 362.00 & 118.68 & 81.32 \\
350 & 276.10 & 110.08 & 57.78 & 576.84 & 444.86 & 207.22 & 142.78 \\
500 & 344.72 & 167.14 & 90.90 & 654.02 & 504.26 & 295.75 & 204.25 \\
1000 & 525.85 & 367.42 & 209.75 & 842.59 & 653.66 & 590.39 & 409.61 \\
5000 & 1332.74 & 2044.96 & 1221.66 & 1733.04 & 1395.13 & 2937.29 & 2062.71 \\
10,000 & 1935.84 & 4011.63 & 2331.14 & 2721.46 & 2305.89 & 5850.21 & 4149.79
\end{tabular}


Baron, Berman, and Perry: Shelf Space Management When Demand Depends on the Inventory Level Production and Operations Management 20(5), pp. 714-726, (C) 2010 Production and Operations Management Society

Table 4 Sensitivity to Different Total Shelf Space: Low-Influence Type

\begin{tabular}{lrrrrrrr}
\hline$S_{T}$ & Profit & \multicolumn{1}{c}{$a_{1}$} & \multicolumn{1}{c}{$a_{2}$} & \multicolumn{1}{c}{$q_{1}$} & \multicolumn{1}{c}{$q_{2}$} & \multicolumn{1}{c}{$S_{1}$} & \multicolumn{1}{c}{$S_{2}$} \\
\hline 50 & 28.90 & 0.49 & 0.01 & 204.96 & 158.00 & 29.67 & 20.33 \\
75 & 34.33 & 1.08 & 0.06 & 223.70 & 171.83 & 44.34 & 30.66 \\
100 & 38.64 & 1.79 & 0.14 & 239.06 & 183.08 & 58.94 & 41.06 \\
150 & 45.37 & 3.37 & 0.41 & 264.42 & 201.96 & 88.00 & 62.00 \\
200 & 50.62 & 5.04 & 0.73 & 286.16 & 217.31 & 116.98 & 83.02 \\
350 & 61.88 & 9.43 & 1.71 & 342.28 & 257.70 & 203.21 & 146.79 \\
500 & 69.67 & 12.60 & 2.49 & 392.31 & 291.91 & 289.11 & 210.89 \\
1000 & 85.50 & 15.46 & 2.86 & 563.89 & 417.78 & 579.35 & 420.65 \\
1500 & 93.13 & 9.78 & 1.11 & 861.59 & 627.51 & 871.38 & 628.63 \\
2000 & 96.51 & 3.81 & 0.11 & 1161.26 & 834.83 & 1165.06 & 834.94 \\
2497 & 97.43 & 0.79 & 0.00 & 1456.57 & 1039.65 & 1457.35 & 1039.65 \\
\hline
\end{tabular}

high demand rate, the higher revenue rate more than offsets the extra holding cost resulting from carrying the additional inventory.

- The percentage of total space that should be allocated to each product, $S_{i} / S_{T}$, is relatively independent of the total shelf space and $S_{1} /$ $S_{T}$ is decreasing in a mild fashion when $S_{T}$ is increasing. Also, the ratio $a_{1} / a_{2}$ is decreasing with $S_{T}$ showing that the operational benefit given to the product with the higher demand is slowly decreasing with the total shelf space allowed.

- the ratio $q_{1} / q_{2}$ shows no clear pattern.

(b) In the low-influence type, the relations are not as clear, because at some $S_{T} \in[500,1000]$ the constraints $S_{i} \leq a_{i}+q_{i}$ become active. We observe:

- For low $S_{T}$, the reorder points are increasing. For high $S_{T}$, above 1000, the reorder points are decreasing.

- The ratio $S_{1} / S_{T}$ is decreasing in a mild fashion until $S_{T} \in[500,1000]$ and increasing from there. The ratio $a_{1} / a_{2}$ is decreasing for $S_{T} \in[50,500]$ and increasing for higher $S_{T}$. Thus, as the constraints $S_{i} \leq a_{i}+q_{i}$ become active, the operational benefit given to the product with the higher demand is increasing.

To investigate the influence of the markup, we varied the markup for the two items, $p_{1}=p_{2}=p$ from 1.3 to 1.4 in steps of 0.02 . We considered the three influence types and $S_{T}=50$ and 200. The results for the medium-influence type and $S_{T}=50$ are reported in Table 5 and are typical. We notice that the order quantities and the shelf space allocation are relatively independent of the markup. However, the reorder
Table 5 Changing the Markup for the Medium-Influence Type When $S_{T}=\mathbf{5 0}$

\begin{tabular}{lrrrcccc}
\hline$p_{1}=p_{2}$ & Profit & \multicolumn{1}{c}{$a_{1}$} & $a_{2}$ & $q_{1}$ & $q_{2}$ & $S_{1}$ & $S_{2}$ \\
\hline 1.3 & 77.29 & 8.57 & 3.00 & 308.47 & 232.48 & 29.84 & 20.16 \\
1.32 & 84.22 & 9.28 & 3.78 & 308.45 & 232.48 & 29.80 & 20.20 \\
1.34 & 91.15 & 10.24 & 4.41 & 308.41 & 232.48 & 29.79 & 20.21 \\
1.36 & 98.08 & 11.08 & 4.96 & 308.37 & 232.47 & 29.77 & 20.23 \\
1.38 & 105.03 & 11.84 & 5.50 & 308.32 & 232.46 & 29.76 & 20.24 \\
1.4 & 111.97 & 12.55 & 6.02 & 308.25 & 232.44 & 29.74 & 20.26 \\
\hline
\end{tabular}

points are increasing with the markup and so does the profit. This is explained by the fact that the higher markup induces the retailer to end the inventory cycles earlier, reducing the period with relatively low demand rate. Again, the higher revenues generated at higher demand rate offset the increase in holding cost resulting from carrying higher inventory levels during the whole cycle. Another observation is that, when the markup price increases from $30 \%$ to $40 \%$, the increase in profit is $44 \%$. In the computation not shown here, this increase ranges between $36 \%$ and $53 \%$.

In another set of experiment, not shown here, we let $\alpha_{01}=2$, and $\alpha_{02}=1$, with $\alpha_{1}=\alpha_{2}=10$, so that product 1 is the product with the higher demand due to a higher intercept rather than dependency on the shelf space and inventory level. We fixed the markup $p_{1}=p_{2}=1.3$ and consider the low-, medium-, and high-influence types, as before. The observations from this set of experiments is similar to the ones reported above. Specifically, $S_{1} / S_{2}$ and $q_{1} / q_{2}$ are both larger than 1 indicating that the seller should use its operational controls to push the higher demand product. Both these ratios decrease toward one as the shelf space increases, indicating that as the constraint imposed by the shelf space is constrained there is less benefit in promoting the higher demand product. While here $a_{1} /$ $a_{2}$ was often smaller than 1 -the demand of the high demand product at the optimal reorder point is still higher than the demand of product 2, further supporting the above conclusion. We note that for the low sensitivity type, the most profitable total shelf space was 1812 units, and this size was higher than 10,000 for the other two sensitivity types.

\subsection{Is Capturing Both Dependencies Important?}

To analyze the importance of capturing both demand dependencies, we considered two heuristics: Heuristic 1 that ignores the inventory dependence and Heuristic 2 that ignores the shelf space dependence. Heuristic 1 is in line with the literature stream focusing on shelf space allocation that ignores the dependency of demand on the inventory level; and Heuristic 2 is in line with the literature focusing on the inventory-dependent demand rate. 

form

Heuristic 1 assumes that the demand rate is of the

$$
r_{i}^{1}(\vec{S})=\alpha_{0 i}^{1}+\alpha_{i}^{1} \Pi_{j \neq i} S_{j}^{\delta_{i j}^{1}}\left(S_{i}^{\beta_{i}^{1}}\right) \quad 0 \leq I_{i}
$$

As seen above, we denote parameters related to this heuristic with a superscript 1 . Heuristic 2, denoted with a superscript 2, assumes that the demand rate is of the form

$$
r_{i}^{2}\left(I_{i}, \vec{S}\right)= \begin{cases}\alpha_{0 i}^{2}+\alpha_{i}^{2} S_{i}^{\beta_{i}^{2}} & I_{i} \geq S_{i} \\ \alpha_{0 i}^{2}+\alpha_{i}^{2} I_{i}^{\beta_{i}} & 0 \leq I_{i}<S_{i} .\end{cases}
$$

In practice, users considering different demand structures will have to estimate different parameters. For discussion on estimating such parameters, see Desmet and Renaudin (1998), Urban (2005), and Balakrishnan et al. (2004).

However, obtaining empirical data to estimate the different parameters of the heuristics is outside the scope of this paper. Still, we wish to obtain some insight about the limitation of the different heuristics. To do so, we search for the best possible parameter choices of the heuristics. We define this best choice as the one that leads to the lowest losses in comparison to the "real" model, given in (22).

Mathematically, we let $\vec{\gamma}_{1}=\left(\alpha_{01}^{1}, \alpha_{1}^{1}, \delta_{12}^{1}, \beta_{1}^{1}, \alpha_{02}^{1}, \alpha_{2}^{1}\right.$, $\left.\delta_{21}^{1}, \beta_{2}^{1}\right)$ denote the vector of parameters for Heuristic 1 . Then, for a given $\vec{\gamma}_{1}$, Heuristic 1 maximizes the long-run profit rate (recall that in the absence of inventory dependence, $a_{1}=a_{2}=0$ )

$$
\begin{aligned}
& \max _{\vec{q}, \vec{S}} \sum_{i=1}^{2} R_{i}^{1}\left(q_{i}, \vec{S} ; \vec{\gamma}_{1}\right), \\
& \sum_{i=1}^{2} S_{i} \leq S_{T} \\
& 0 \leq S_{i} \leq q_{I} \quad \forall i=1,2
\end{aligned}
$$

where with $E\left(T_{i}^{1}\right)=q_{i} / r_{i}^{1}(\vec{S})$ and $E\left(H_{i}^{1}\right) / E\left(T_{i}^{1}\right)=h_{i} q_{i} / 2$ we have

$$
R_{i}^{1}\left(q_{i}, \vec{S} ; \vec{\gamma}_{1}\right)=\frac{c_{i}\left(p_{i}-1\right) q_{i}-E\left(H_{i}^{1}\right)-K_{i}}{E\left(T_{i}^{1}\right)}+c_{s_{i}} S_{i} .
$$

For a given $\vec{\gamma}_{1}$, we denote the optimal decisions recommended by Heuristic 1 by $q_{1}^{* 1}\left(\vec{\gamma}_{1}\right), q_{2}^{* 1}\left(\vec{\gamma}_{1}\right), S_{1}^{* 1}\left(\vec{\gamma}_{1}\right)$, and $S_{2}^{* 1}\left(\vec{\gamma}_{1}\right)$. With this choice of control variables, the profit realized in the real model would be $R^{* 1}\left(\vec{\gamma}_{1}\right) \equiv$ $\sum_{i=1}^{2} R_{i}\left(0, q_{i}^{* 1}\left(\vec{\gamma}_{1}\right), \vec{S}^{* 1}\left(\vec{\gamma}_{1}\right)\right)$ where $R_{i}\left(0, q_{i}, \vec{S}\right)$ is given in (5).

Let $R^{*}$ denote the optimal profit in the real model. Then, for Heuristic 1 and any given $S_{T}$ we look for the parameters vector $\vec{\gamma}_{1}$ that solves

$$
\arg \min _{\vec{\gamma}_{1}} R^{*}-R^{* 1}\left(\vec{\gamma}_{1}\right)=\arg \max _{\vec{\gamma}_{1}} R^{* 1}\left(\vec{\gamma}_{1}\right) .
$$

The ratio $L_{1} \equiv\left(R^{*}-\max _{\vec{\gamma}_{1}} R^{* 1}\left(\vec{\gamma}_{1}\right)\right) / R^{*}$ provides a lower bound on the losses of a retailer that ignores the demand dependency on the inventory level, i.e., uses Heuristic 1 rather than the real model.

We follow a similar procedure to provide $L_{2}$, a lower bound for a retailer that uses Heuristic 2 . Observe that finding both $L_{1}$ and $L_{2}$ involves solving infinite many optimization problems similar to (23). Thus, the computation effort to estimate these bounds might be very demanding.

Luckily, for Heuristic 2, we show:

Proposition 2. $L_{2}=0$

Proof. Consider the "real world's" demand rate given in (14) and any optimal choice $\vec{S}^{*}$. Then, for Heuristic 2 one can choose

$$
\begin{aligned}
& \alpha_{0 i}^{2}=\alpha_{0 i}+\alpha_{1 i}\left(S_{i}^{*}\right)^{\beta_{1 i}}\left(S_{j}^{*}\right)^{\delta_{1 i j}}, \\
& \beta_{2 i}^{2}=\beta_{2 i}, \\
& \alpha_{i}^{2}=\alpha_{2 i}\left(S_{j}^{*}\right)^{\delta_{2 i j}},
\end{aligned}
$$

which causes this heuristic to estimate a demand rate that is identical to the "real world's" one. This will give rise to optimal decisions.

Note that the value of the "optimal" $\alpha$ 's parameters chosen in Proposition 2 is substantially different than the "real world" ones. Thus, it might not be realistic to assume that this value will be found in practice. Still, this proposition prevents us from concluding any negative results on the cost of assuming that demand is independent of the shelf space when it actually does.

Thus, we only search for $L_{1}$. For this we assume that the real demand rate for the item is given in (22). We considered the medium-influence type and present the results for the predicted profit of Heuristic $1, H^{1 *}\left(\vec{\gamma}_{1}\right), L_{1}$, and $\max _{\vec{\gamma}_{1}} R^{* 1}\left(\vec{\gamma}_{1}\right)$ as a function of $S_{T} \in\{50,75,100,150,200,350,500,1000,5000,10,000\}$ in Table 6.

We searched for the $\vec{\gamma}_{1}$ that minimizes $L_{1}$ as follows: We initialized the search with $\vec{\gamma}_{1}$ values $\alpha_{01}^{1}=\alpha_{02}^{1}=1$, $\beta_{1}^{1}=\beta_{2}^{1}=0.5, \delta_{12}^{1}=\delta_{21}^{1}=0.1$, as in the real model, and $\alpha_{1}^{1}=30, \alpha_{2}^{1}=20$, i.e., letting $\alpha_{1}^{1}$ and $\alpha_{2}^{1}$ be twice the value of the real model if the inventory never drops below the shelf space level. This initial point overestimates the real demand whenever the inventory level is below the allocated shelf space for the product. We then ran an exhaustive search around this point until we could find no improvement or when we identified a run with $L_{1}<0.1 \%$. We set the step sizes for this search to 1 for $\alpha_{01}^{1}, \alpha_{02}^{1}, \alpha_{1}^{1}$, and $\alpha_{2}^{1}$, and to 0.05 for $\beta_{1}^{1}, \beta_{2}^{1}$, $\delta_{12}^{1}$, and $\delta_{21}^{1}$. 
Table 6 The Predicted Profit, the Lower Bound, and Actual Profit of Heuristic 1 When $S_{T}$ Varies for the Medium-Influence Type

\begin{tabular}{lrcr}
\hline$S_{T}$ & $H^{* 1}\left(\vec{\gamma}_{1}^{*}\right)$ & $L_{1}$ in $\%$ & $\max _{\vec{\gamma}_{1}} R^{* 1}\left(\vec{\gamma}_{1}\right)$ \\
\hline 50 & 88.37 & 0.396 & 77.29 \\
75 & 127.31 & 0.617 & 101.79 \\
100 & 161.38 & 0.869 & 123.30 \\
150 & 246.69 & 1.244 & 160.83 \\
200 & 328.91 & 1.593 & 193.64 \\
350 & 614.42 & 2.413 & 276.10 \\
500 & 949.68 & 3.011 & 344.72 \\
1000 & 2361.43 & 4.230 & 525.85 \\
5000 & $24,851.94$ & 6.058 & 1332.74 \\
10,000 & $72,250.97$ & 5.694 & 1935.84 \\
\hline
\end{tabular}

From Table 6, we notice that the profits realized using the controls recommended by the heuristic are lower than the profits it predicts and that the relative error of Heuristic 1 in profits from the optimal policy is increasing with $S_{T}$ from less than $1 \%$ for low $S_{T}$ to about $6 \%$ for high $S_{T}$. We thus conclude that, when the total shelf space is very limited, it might be safe to ignore the demand dependency on the inventory level. However, ignoring this dependency when the shelf space is not as limited is likely to cause significant losses.

This observation can be explained by that when $S_{T}$ is very limited, the relative duration of time when the inventory level is below the shelf space is shorter and thus it is less risky to ignore it.

From a comparison of the losses for the same $S_{T}$ values for the low and high demand influence types (not reported here), we see, as expected, that ignoring the demand dependency on the inventory level is more costly when the demand is more sensitive to this dependency.

Both demand dependencies on shelf space and on the inventory level have won plenty of attention in leading academic journals. The results of this subsection, that $L_{2}=0$ and $L_{1}$ might be larger than $6 \%$, suggest that models that consider only the dependency on the inventory level might perform better in practice (than models that consider only the dependency on shelf space). Moreover, models that consider only the dependence on shelf space may result in suboptimal decisions, especially when the shelf space is high as in Home Depot, e.g., Balakrishnan et al. (2004).

\subsection{On Ignoring the Intercept}

To demonstrate the importance of including the intercept in modeling the demand rate, we present an example. We consider the medium influence demand rate from our examples above with $S_{T}=50$. We solved for the optimal decisions of a model that assumes that the demand rate has no intercept, i.e., it is of the form

$$
r_{i}^{3}\left(I_{i}, \vec{S}\right)= \begin{cases}\alpha_{i}^{3} \Pi_{j \neq i} S_{j}^{\delta_{i j}^{3}}\left(S_{i}^{\beta_{i}^{3}}+S_{i}^{\beta_{i}^{3}}\right) & I_{i} \geq S_{i} \\ \alpha_{i}^{3} \Pi_{j \neq i} S_{j}^{\delta_{i j}^{3}}\left(S_{i}^{\beta_{i}^{3}}+I_{i}^{\beta_{i}^{3}}\right) & 0 \leq I_{i}<S_{i} .\end{cases}
$$

As seen above, we denote parameters related to this model with a superscript 3 .

As in the subsection above, we looked for a lower bound on the losses of a retailer that ignores the intercepts. We define $L_{3}$ similar to the definition of $L_{1}$ and $L_{2}$. A similar exhaustive search over the parameters of this model leads to $L_{3}=80.72 \%$ with an actual profit of US\$14.90 rather than US\$77.29 in the real model. Similar to what we observed when investigating $L_{2}$ the profit predicted by the model was much higher (US\$298.03).

Note that the intercept for both products in our example are $\alpha_{01}=\alpha_{02}=1$, which are an order of magnitude smaller than $\alpha_{1}=15$ and $\alpha_{2}=10$. Thus, we expect that for relatively larger intercepts $L_{3}$ would be even higher. This example emphasizes the importance of including the intercepts in the demand rate model. It also supports our inventory domain approach that enables including intercepts in the demand rates.

\section{Conclusion and Future Research}

In this paper, we investigated the joint shelf space allocation and inventory control problem for multiple products with demand that depends on both the inventory level seen by customers and on the shelf space allocated to the product and to its complement or supplement products. We analyze the problem in the inventory domain via level crossing theory. This allowed us to obtain closed-form expressions for the profit rate for a large family of demand rate functions exhibiting both dependencies. We showed that using these expressions one can solve the joint control problem. Using an extensive numerical study with two products, we analyzed the sensitivity of optimal decision variables and profit to different parameters of the demand. We derived several interesting managerial insights. They include (a) that in some settings it is important to capture both demand dependencies and that ignoring the dependency on the shelf space might be less harmful than ignoring the dependency on the inventory level. The latter can lead to profit losses of more than 6\%; (b) that ignoring the intercepts in the demand model might be very costly; and (c) that retailers should use their operational controls (e.g., reorder point) to promote higher demand products.

It is important to note that the inventory domain methodology may be used to investigate additional 
settings where the time domain approach fails. This can be an interesting future research direction. Another important extension of this work would be to estimate the demand rates for products using empirical data. This might also help to investigate the actual performance of the heuristics ignoring either one of the dependencies rather than using a lower bound on their performance. Finally, there might be a need to develop efficient algorithms for solving similar joint optimization problems in the presence of many items, such as in Lim et al. (2004).

\section{Acknowledgments}

This paper was supported by NSERC grants of the first two authors. We thank the referees and associate editor for their comments.

\section{Appendix A. Proof of Theorem 1}

When yield is random and the amount ordered is $q$, the amount supplied is the random variable $Y_{q}$. For every $I>0$ we define $U_{I}(t)$ and $D_{I}(t)$ as the number of up- and downcrossings of level $I$ until $t$ by $I(t, S)$, respectively. Observe that for every $t>0$, we have $\left|D_{I}(t)-U_{I}(t)\right| \leq 1$ no matter what the starting point is (see the intuitive explanation above). Therefore, $\lim _{t \rightarrow \infty}(1 / t)\left|D_{I}(t)-U_{I}(t)\right|=0$. The latter argument simply says that the long-run average number of downcrossings $d_{I} \equiv \lim _{t \rightarrow \infty}(1 / t) D_{I}(t)$ must equal the long-run average number of upcrossings $u_{I} \equiv$ $\lim _{t \rightarrow \infty}(1 / t) U_{I}(t)$ for any level $I \geq a>0$. Thus, $d_{I}=u_{I}$. Furthermore, given the regenerative structure of the inventory level and assuming that at time $t=0 \mathrm{a}$ regeneration cycle starts,

$$
D_{I}(T)=U_{I}(T)=1_{\left\{I \in\left(a, a+Y_{q}\right)\right\}},
$$

where $T$ is the cycle length and $1_{\{\cdot\}}$ is the indicator function. Also, by the Renewal Reward Theorem

$$
d_{I}=\frac{E\left[D_{I}(T)\right]}{E(T)}=\frac{E\left[U_{I}(T)\right]}{E(T)}=u_{I}
$$

We observe that the time the content level process $I(t, S)$ stays in $(I, I+\Delta I]$ during a cycle $T$ for small $\Delta I$ is

$$
\frac{\Delta I D_{I}(T)}{r(I, S)} \approx \int_{0}^{T} 1_{\{I<I(t, S) \leq I+\Delta I\}} d t .
$$

Moreover, because $D_{I}(T)$ is either 0 or 1 we can take the limit as $\Delta I \rightarrow 0$ (by dominated convergence) to obtain

$$
\begin{aligned}
D_{I}(T) & =r(I, S) \lim _{\Delta I \rightarrow 0} \frac{\int_{0}^{T} 1_{\{I<I(t, S) \leq I+\Delta I\}} d t}{\Delta I} \\
& \equiv r(I, S) \frac{d}{d I} \int_{0}^{T} 1_{\{I(t, S) \leq I\}} d t .
\end{aligned}
$$

Taking expectation in (A2) we obtain for each $I \leq a+q$

$$
\begin{aligned}
E\left(D_{I}(T)\right) & =r(I, S) E\left(\frac{d}{d I} \int_{0}^{T} 1_{\{I(t, S) \leq I\}} d t\right) \\
& =r(I, S) E(T) \frac{d}{d I} \frac{E\left(\int_{0}^{T} 1_{\{I(t, S) \leq I\}} d t\right)}{E(T)},
\end{aligned}
$$

where the second equality follows dominated convergence.

Let $F(I)$ be the steady-state distribution of $I(t, S)$. If $F(I)$ is an absolutely continuous distribution, $f(I) \Delta I$ represents approximately the long-run average proportion of time the inventory level process stays at the neighborhood of $I$ and $f(I)$ represents the steady-state density. Moreover, this is the exact interpretation for the element in the derivative in (A3). We then obtain

$$
E\left(D_{I}(T)\right)=r(I, S) E(T) f(I) .
$$

We further observe that the content level process during a cycle is bounded between $a$ and $a+Y_{q}$ implying that the expected number of upcrossings of level $I$ during a cycle is

$$
\begin{aligned}
E\left(U_{I}(T)\right) & =E\left(1_{\left\{Y_{q}>I-a\right\}}\right)=P\left(Y_{q}>I-a\right) \\
& \equiv 1-F_{Y_{q}}(I-a) .
\end{aligned}
$$

Finally, (A1) implies that the left-hand sides of (A5) and (A4) are equal. Thus, so are their right-hand sides and, when ordering at $a \geq 0$, we obtain (15). The expressions for (16) and (18) follow immediately, completing the proof.

We note that when $F(I)$ is not an absolutely continuous distribution the derivative $\left(\frac{d}{d l} 1_{\{I(t, S) \leq I\}}\right)$ exists only at points of continuity of $F(I)$. More precisely, as $1_{\{I(t, S) \leq I\}} \leq 1$ both the limit from the left and the limit from the right $\lim _{\Delta I \rightarrow 0} E\left(1_{\{I<I(t, s) \leq I+\Delta I\}}\right) / \Delta I$ exist, but might be different such that $f(I+) \neq f(I-)$. Observe that $f(I-) \Delta I \rightarrow 0$ as $\Delta I \rightarrow 0$. Thus, we use arbitrarily the criterion $f(I)=f(I+)$ for all $I \geq a$ and (17) is valid even when $F(I)$ is not an absolutely continuous distribution. Also, because the sample path of $I(t, S)$ decreases during a cycle (between jumps of delivery), when $F_{Y_{q}}$ $(I)$ is absolutely continuous so is $F(I)$.

\section{Note}

${ }^{1}$ As in Balakrishnan et al. (2004), we can treat a more general demand by further defining $\phi_{1 i}, \phi_{1 i j}, j \neq i$, and $\phi_{2 i}, \phi_{2 i j}, j \neq i$, to represent a base shelf space for the shelf space dependencies' effects on item $i$ measured via its allocated shelf space, and inventory level, respectively. Then the demand rate function would be

$$
\begin{aligned}
& r_{i}\left(I_{i}, \vec{S}\right) \\
& = \begin{cases}\alpha_{0 i}+\alpha_{1 i}\left(S_{i}+\phi_{1 i}\right)^{\beta_{1 i}} \Pi_{j \neq i}\left(S_{j}+\phi_{1 i j}\right)^{\delta_{1 i j}}+\alpha_{2 i} S_{i}^{\beta_{2}} \Pi_{j \neq i}\left(S_{j}+\phi_{2 i j}\right)^{\delta_{2 i j}} & I_{i} \geq S_{i} \\
\alpha_{0 i}+\alpha_{1 i}\left(S_{i}+\phi_{1 i}\right)^{\beta_{1 i}} \Pi_{j \neq i}\left(S_{j}+\phi_{1 i j}\right)^{\delta_{1 i j}}+\alpha_{2 i} i_{i}^{\beta_{2}} \Pi_{j \neq i}\left(S_{j}+\phi_{2 i j}\right)^{\delta_{2 i j}} & 0 \leq I_{i}<S_{i} .\end{cases}
\end{aligned}
$$

We believe the extension in (14) should suffice in most cases. 
Baron, Berman, and Perry: Shelf Space Management When Demand Depends on the Inventory Level Production and Operations Management 20(5), pp. 714-726, (C) 2010 Production and Operations Management Society

\section{References}

Akcay, Y., B. Tan. 2008. On the benefits of assortment-based cooperation among independent producers. Prod. Oper. Manag. 17(6): 626-640.

Aydin, G., W. H. Hausman. 2009. The role of slotting fees in the coordination of assortment decisions. Prod. Oper. Manag. 17(6): 626-640.

Baker, R. C., T. L. Urban. 1988. A deterministic inventory system with inventory-level-dependent demand rate. J. Oper. Res. Soc. 39: 823-825.

Balakrishnan, A., M. S. Pangburn, E. Stavrulaki. 2004. Stack them high, let 'em fly: Lot-sizing policies when inventories stimulate demand. Manage. Sci. 50(5): 630-644.

Balakrishnan, A., M. S. Pangburn, E. Stavrulaki. 2005. Integrating the promotional and service roles of retail inventories. McCombs research paper series no. IROM-02-05. Available at SSRN http:/ / ssrn.com/abstract=759948 (accessed November 5, 2010).

Berman, O., D. Perry. 2006. An EOQ model with state-dependent demand rates. Eur. J. Oper. Res. 171(1): 255-272.

Bookbinder, J. H., F. H. Zarour. 2001. Direct product profitability and retail shelf-space allocation models. J. Bus. Logist. 22(2): 183-207.

Borin, N., P. W. Farris, J. R. Freeland. 1994. A model for determining retail product category assortment and shelf space allocation. Decis. Sci. 25(3): 359-384.

Bultez, A., P. Naert. 1988. SH.A.R.P.: Shelf allocation for retailer's profit. Mark. Sci. 7(3): 211-231.

Cohen, J. W. 1977. On up- and downcrossing. J. Appl. Probab. 14: 405-410.

Corstjens, M., P. Doyle. 1981. A model for optimizing retail space allocations. Manage. Sci. 27(7): 822-833.

Datta, T. K., A. K. Pal. 1990. A note on an inventory model with inventory-level-dependent demand rate. J. Oper. Res. Soc. 41: 971-975.

Desmet, P., V. Renaudin. 1998. Estimation of product category sales responsiveness to allocated shelf space. Int. J. Res. Mark. 15: 443-457.

Hariga, M. A., A. Al-Ahmari, A. A. Mohamed. 2007. A joint optimisation model for inventory replenishment, product assortment, shelf space and display area allocation decisions. Eur. J. Oper. Res. 181(1): 239-251.
Henig, M., Y. Gerchak. 1988. The structure of periodic review policies in the presence of variable yield. IIE Trans. 20: 144-150.

Hwang, H., B. Choi, M. Lee. 2005. A model for shelf space allocation and inventory control considering location and inventory level effects on demand. Int. J. Prod. Econ. 97: 185-195.

Gerchak, Y., Y. Wang. 1994. Periodic-review inventory models with inventory-level-dependent demand. Nav. Res. Logisit. 41: 99-116.

Lee, W. 1961. Space management in retail stores and implications to agriculture. Dolva, W. K. ed. Marketing Key's to Profit in the 1960's. American Marketing Association, Chicago, 523-533.

Lim, A., B. Rodriguees, X. Zhang. 2004. Metaheuristics with local search techniques for retail shelf-space optimization. Manage. Sci. 50(1): 117-131.

Martinez-de-Albeniz, V., G. Roels. 2010. Competing for shelf space. Prod. Oper. Manag. (forthcoming).

Parker, R. J., R. D. Lehmann. 2009. The process underlying the bandwagon effect. Working paper, Columbia Business School, Columbia University.

Smith, A. S., D. D. Achabal. 1998. Clearance pricing and inventory policies for retail chains. Manage. Sci. 44(3): 285-300.

Ton, Z., A. Raman. 2010. The effect of product variety and inventory levels on retail store sales. Prod. Oper. Manag. 19(5): 546-560.

Urban, T. L. 2005. Inventory models with inventory-level-dependent demand: A comprehensive review and unifying theory. Eur. J. Oper. Res. 162: 792-804.

van Herpen, E., R. Pieters, M. Zeelenberg. 2009. When demand accelerates demand: Trailing the bandwagon. J. Consum. Psychol. 19: 302-312.

van Nierop, E., D. Fok, P. H. Franses. 2008. Interaction between shelf layout and marketing effectiveness and its impact on optimizing shelf arrangements. Mark. Sci. 27(6): 1065-1082.

Wang, Y., Y. Gerchak. 2001. Supply chain coordination when demand is shelf space dependent. Manuf. Serv. Oper. Manag. 3(1): 82-87.

Whitin, T. M. 1957. The Theory of Inventory Management. Princeton University Press, Princeton, NJ.

Wolfe, H. B. 1968. A model for control of style merchandise. Ind. Manage. Rev. (Sloan Manage. Rev.) 9(2): 69-82.

Zhaolin, L. 2007. A single-period assortment optimization model. Prod. Oper. Manag. 16(3): 369-380. 